\title{
On packing of two copies of a hypergraph
}

\author{
Monika Pilśniak\| and Mariusz Woźniak \\ AGH University of Science and Technology, Department of Discrete Mathematics, Kraków, Poland
}

A 2-packing of a hypergraph $\mathcal{H}$ is a permutation $\sigma$ on $V(\mathcal{H})$ such that if an edge $e$ belongs to $\mathcal{E}(\mathcal{H})$, then $\sigma(e)$ does not belong to $\mathcal{E}(\mathcal{H})$. Let $\mathcal{H}$ be a hypergraph of order $n$ which contains edges of cardinality at least 2 and at most $n-2$. We prove that if $\mathcal{H}$ has at most $n-2$ edges then it is 2-packable.

Keywords: packing, hypergraphs

\section{Introduction}

Let $\mathcal{H}=(V, \mathcal{E})$ be a hypergraph, where $V$ is the vertex set and $\mathcal{E} \subset 2^{V}$ is the edge set. We allow empty edges for technical reasons, hence a complete simple hypergraph of order $n$ has $2^{n}$ edges. We consider only finite hypergraphs. The edge of cardinality $t$ is called $t$-edge, and 1-edge is called a singleton. A vertex is isolated if it does not belong to any edge. The number $d(v)$ of edges containing a vertex $v$ is called the degree of $v \in V$. A hypergraph is $t$-uniform if $|e|=t$ for all $e \in \mathcal{E}$. Let $\mathcal{H}$ be a hypergraph of order $n$. A packing of two copies of $\mathcal{H}$ (2-packing of $\mathcal{H}$ ) is a permutation $\sigma$ on $V(\mathcal{H})$ such that, if an edge $e=\left\{x_{1}, \ldots, x_{k}\right\}$ belongs to $\mathcal{E}(\mathcal{H})$, then the edge $\sigma(e)=\left\{\sigma\left(x_{1}\right), \ldots, \sigma\left(x_{k}\right)\right\}$ does not belong to $\mathcal{E}(\mathcal{H})$. Such a permutation (a packing permutation) is also called an embedding of $\mathcal{H}$ into its complement. Consider a hypergraph $\mathcal{H}$ and a permutation $\sigma$ on $V$. We have $\sigma(V)=V$ and $\sigma(\emptyset)=\emptyset$. So, if $V \in \mathcal{E}$ or $\emptyset \in \mathcal{E}$, then $\mathcal{H}$ cannot be packable.

We proved the following result in [4].

Theorem 1 If a hypergraph $\mathcal{H}$ of order $n$ and size at most $\frac{1}{2} n$ has neither the empty edge nor its complement, then $\mathcal{H}$ is 2-packable.

Observe that this bound is sharp. Namely, if $\mathcal{H}$ is a hypergraph of order $n$, and it has more than $\frac{1}{2} n$ edges, and each edge is a singleton, then evidently $\mathcal{H}$ is not packable.

The aim of this paper is to show that if empty edges and singletons (and their complements, i.e. $n$-edges and $(n-1)$-edges) are excluded, then the bound on the size can be improved. We call a hypergraph $\mathcal{H}$ of order $n$ admissible if $2 \leq|H| \leq n-2$ holds for all edges $H \in \mathcal{H}$.

We shall prove the following theorem.

\footnotetext{
$\dagger$ Email: pilsniakeagh.edu.pl

‡Email: mwozniakeagh.edu.pl

This research was partially supported by the Polish Ministry of Science and Higher Education

1365-8050 @ 2011 Discrete Mathematics and Theoretical Computer Science (DMTCS), Nancy, France
} 
Theorem 2 An admissible hypergraph $\mathcal{H}$ of order $n$ and size at most $n-2$ is 2-packable.

Recall that a 2-uniform hypergraph is called a graph. The packing problems for graphs have been studied for about thirty years (see for instance chapters in the books by B. Bollobás or H. P. Yap ([2],[8]), or survey papers by H.P. Yap or M. Woźniak ([9], [6], [7] and [5])). One of the first results in this area was the following theorem (see [3]).

Theorem 3 A graph $G$ of order $n$ and size at most $n-2$ is 2-packable.

This bound is tight. Namely, if $G$ is a star (of order $n$ and size $n-1$ ), then $G$ is not packable.

Let $\mathcal{H}$ be an admissible hypergraph of order $n$. First, denote by $\mathcal{H}_{k}$ a $k$-uniform hypergraph of order $n$, which is induced by all $k$-edges in $\mathcal{H}$, and let $m_{k}$ be the size of $\mathcal{H}_{k}$. Let $m$ be the size of $\mathcal{H}$. Thus

$$
n-2 \geq m=m_{2}+m_{3}+\ldots+m_{n-2} .
$$

Let $\mathcal{H}=(V, \mathcal{E})$ be a hypergraph. Consider the hypergraph $\tilde{\mathcal{H}}=(V, \tilde{\mathcal{E}})$ with the same vertex set $V$ and the edge set $\tilde{\mathcal{E}}$, obtained from $\mathcal{E}$ in the following way: if $e \in \mathcal{E}$ has at most $\frac{n}{2}$ vertices then $e$ belongs to $\tilde{\mathcal{E}}$ and if $e$ has more than $\frac{n}{2}$ vertices, then $e$ is replaced by $V \backslash e$, with the convention that a double edge conceivably created in this way is replaced by a single one.

Remark 4 Let $\mathcal{H}$ be an admissible hypergraph of order $n$. If the hypergraph $\tilde{\mathcal{H}}$ is 2-packable, then also $\mathcal{H}$ is 2-packable. Therefore, we shall assume that $\mathcal{H}$ of order $n$ is restricted to have edges of size at most $n / 2$ only.

Let $\mathcal{H}=(V, \mathcal{E})$ be an admissible hypergraph, and let $x$ be a vertex of $\mathcal{H}$. We define the hypergraph $\mathcal{H}^{\prime}=\left(V^{\prime}, \mathcal{E}^{\prime}\right):=\mathcal{H}-x$ as follows: $V^{\prime}=V \backslash\{x\}$, and the set of edges is obtained from $\mathcal{E}$ by deleting 2-edges containing $x$, and replacing all remaining edges containing $x$ by new edges with $x$ deleted. It should be noted that it may happen that the assumption of Remark 4 does not apply to the hypergraph $\tilde{\mathcal{H}}$. So, if necessary, we use $\tilde{\mathcal{H}}^{\prime}$ instead of $\mathcal{H}^{\prime}$.

\section{Lemmas}

In the proof of Theorem 2, we shall use the following lemmas.

Lemma 5 Let $\mathcal{H}$ be an admissible hypergraph of order $n \geq 7$. Let $x$ be an isolated vertex in $\mathcal{H}_{2}$, and let $y$ be a vertex of degree at least two in $\mathcal{H}_{2}$. Suppose that there does not exist any 3-edge e $\in \mathcal{H}$ such that $x \in e$ and $y \in e$. If $\mathcal{H}^{\prime}=\mathcal{H}-x-y$ is 2-packable, then $\mathcal{H}$ is also 2-packable. Moreover, $\mathcal{H}^{\prime}$ is an admissible hypergraph.

Proof: Let $x$ and $y$ be two vertices satisfying the assumptions. It is easy to see that $\mathcal{H}^{\prime}$ is an admissible hypergraph, since, by assumptions, there is no singleton in $\mathcal{H}^{\prime}$, because there is no 3-edge $e \in \mathcal{H}$ such that $x \in e$ and $y \in e$. On the other hand, since $n \geq 7$, there is no $\left(n^{\prime}-1\right)$-edge in $\mathcal{H}^{\prime}$ (where $n^{\prime}=n-2$ ).

Let $\sigma^{\prime}$ be a packing permutation of $\mathcal{H}^{\prime}$. By the choice of $x$ and $y$ and the property of $\sigma^{\prime}$, it is easy to see that the permutation $\sigma=\sigma^{\prime} \circ(x y)$, where $(x y)$ denotes a transposition, is a packing permutation of $\mathcal{H}$.

The proof of Lemma 6 is analogous to that of Lemma 5 . 
Lemma 6 Let $\mathcal{H}$ be an admissible hypergraph of order $n \geq 7$. Let $x$ and $y$ be two not adjacent vertices of degree one in $\mathcal{H}_{2}$ such that the neighbors $x^{\prime}$ of $x$ and $y^{\prime}$ of $y$ are distinct. Suppose that there does not exist any 3-edge $e \in \mathcal{H}$ such that $x \in e$ and $y \in e$. If $\mathcal{H}^{\prime}=\mathcal{H}-x-y$ is 2-packable, then $\mathcal{H}$ is also 2-packable. Moreover, $\mathcal{H}^{\prime}$ is an admissible hypergraph.

Lemma 7 Let $\mathcal{H}$ be an admissible hypergraph of order $n$ and size at most $n-2$. If $m_{2} \leq \frac{n}{2}$, then $\mathcal{H}$ is 2-packable.

Proof: Using a probabilistic argument we shall show that a packing permutation exists for $\mathcal{H}$.

Let $e$ and $f$ be two edges of $\mathcal{H}$ of the same cardinality and let $\sigma$ be a random permutation on $V$. We say that edge $e$ covers edge $f$ (with respect to $\sigma$ ), if $\sigma(e)=f$. We denote this fact by $(e \curvearrowright f)$.

Let $e$ and $f$ be two $k$-edges. The event $A$ such that $e$ covers $f$ (denoted by $A(e \curvearrowright f)$ ) has probability equal to

$$
\operatorname{Pr}(A(e \curvearrowright f))=\frac{k !(n-k) !}{n !}=\left(\begin{array}{l}
n \\
k
\end{array}\right)^{-1} .
$$

Observe, that there are $m_{k}^{2}$ ways to choose a pair $e, f$ of $k$-edges such that $e$ covers $f$. So, we have

$\operatorname{Pr}\left(\bigcup_{e, f \in \mathcal{H}} A(e \curvearrowright f)\right) \leq \sum_{e, f \in \mathcal{H}} \operatorname{Pr}(A(e \curvearrowright f))=m_{2}^{2}\left(\begin{array}{c}n \\ 2\end{array}\right)^{-1}+m_{3}^{2}\left(\begin{array}{c}n \\ 3\end{array}\right)^{-1}+\ldots+m_{\left\lfloor\frac{n}{2}\right\rfloor}^{2}\left(\begin{array}{c}n \\ \left\lfloor\frac{n}{2}\right\rfloor\end{array}\right)^{-1}$

Since $k \leq \frac{n}{2}$, the sequence $\left(\left(\begin{array}{l}n \\ 2\end{array}\right)^{-1},\left(\begin{array}{l}n \\ 3\end{array}\right)^{-1}, \ldots\right)$ is decreasing, and we have

$$
\begin{gathered}
m_{2}^{2}\left(\begin{array}{c}
n \\
2
\end{array}\right)^{-1}+m_{3}^{2}\left(\begin{array}{l}
n \\
3
\end{array}\right)^{-1}+\ldots+m_{\left\lfloor\frac{n}{2}\right\rfloor}^{2}\left(\begin{array}{c}
n \\
\left\lfloor\frac{n}{2}\right\rfloor
\end{array}\right)^{-1} \leq m_{2}^{2}\left(\begin{array}{c}
n \\
2
\end{array}\right)^{-1}+\left(\begin{array}{c}
n \\
3
\end{array}\right)^{-1}\left(m_{3}^{2}+\ldots+m_{\left\lfloor\frac{n}{2}\right\rfloor}^{2}\right) \leq \\
\leq m_{2}^{2}\left(\begin{array}{c}
n \\
2
\end{array}\right)^{-1}+\left(\begin{array}{c}
n \\
3
\end{array}\right)^{-1}\left(n-2-m_{2}\right)^{2} .
\end{gathered}
$$

If $m_{2}=0$, then $n \geq 5$, and

$$
m_{2}^{2}\left(\begin{array}{l}
n \\
2
\end{array}\right)^{-1}+\left(\begin{array}{l}
n \\
3
\end{array}\right)^{-1}\left(n-2-m_{2}\right)^{2}=\left(\begin{array}{l}
n \\
3
\end{array}\right)^{-1}(n-2)^{2} .
$$

If $m_{2}=1$, then $n \geq 3$, and

$$
m_{2}^{2}\left(\begin{array}{l}
n \\
2
\end{array}\right)^{-1}+\left(\begin{array}{l}
n \\
3
\end{array}\right)^{-1}\left(n-2-m_{2}\right)^{2}=\left(\begin{array}{l}
n \\
2
\end{array}\right)^{-1}+\left(\begin{array}{l}
n \\
3
\end{array}\right)^{-1}(n-3)^{2} .
$$

If $m_{2} \geq 2$, then $n \geq 4$, and

$$
m_{2}^{2}\left(\begin{array}{l}
n \\
2
\end{array}\right)^{-1}+\left(\begin{array}{l}
n \\
3
\end{array}\right)^{-1}\left(n-2-m_{2}\right)^{2} \leq \frac{2 n^{2}}{4 n(n-1)}+\frac{6(n-4)^{2}}{n(n-1)(n-2)} .
$$


It is easy to check that in each case

$$
\operatorname{Pr}\left(\bigcup_{e, f \in \mathcal{H}} A(e \curvearrowright f)\right)<1 .
$$

Consequently, a 2-packing of an admissible hypergraph $\mathcal{H}$ of order $n$ and size at most $n-2$ exists, if $m_{2} \leq \frac{n}{2}$.

\section{Proof of Theorem 2}

By Remark 4, we consider only hypergraphs with edges of cardinality at most $\frac{n}{2}$. It is easy to see that for $n \leq 6$, either $\mathcal{H}$ has only 2-edges, and we can apply Theorem 3 , or the number of 2-edges is less than or equal to $n / 2$, and we can apply Lemma 7 . So, let $n \geq 7$.

Observe that, by Lemma 7, our claim holds if $\mathcal{H}_{2}$ is empty. Therefore, the proof will be divided into two main cases corresponding to the structure of $\mathcal{H}_{2}$ which is supposed to be non-empty.

The proof goes by induction on $n$. Let $x, y$ be two vertices satisfying the assumptions of Lemma 5 or of Lemma 6 A 3-edge containing both of them will be called a blocking edge. Observe that if there is no blocking edge in $\mathcal{H}$, then the induction hypothesis can be applied. Below, we shall very often estimate the number of blocking edges in order to get a contradiction with the size of $\mathcal{H}$.

Case 1. There is no vertex of degree one in $\mathcal{H}_{2}$.

The hypergraph $\mathcal{H}_{2}$ has at most $n-2$ edges, so it has at least two isolated vertices. Denote by $w$ the number of non-isolated vertices in $\mathcal{H}_{2}$. Observe that $w \geq 3$ and $w \leq m_{2}$. Let $y$ be a vertex of degree at least 2 in $\mathcal{H}_{2}$. If we can choose an isolated vertex $x$ in $\mathcal{H}_{2}$ such that there is no 3 -edge containing both $x$ and $y$, then we are done. So, suppose that a 3 -edge containing both $x$ and $y$ exists in $\mathcal{H}$ for every isolated vertex $x$ in $\mathcal{H}_{2}$ and for any $y$. Observe that one 3 -edge can cover at most two pairs of vertices $x, y$ satisfying the assumptions of Lemma 5 . Hence,

$$
\begin{gathered}
m_{3} \geq \frac{1}{2} w(n-w) \geq \frac{1}{2} w\left(n-m_{2}\right), \\
2 m_{3}+w m_{2} \geq w n .
\end{gathered}
$$

Hence,

$$
w\left(m_{2}+m_{3}\right) \geq w n,
$$

but $m_{2}+m_{3} \leq n-2$, a contradiction.

Case 2. There is a vertex of degree one in $\mathcal{H}_{2}$.

Let $b=m_{3}+\ldots+m_{\left\lfloor\frac{n}{2}\right\rfloor}$. If $b=0$, then $\mathcal{H}$ is a graph, and the claim is true. Hence, let $b>0$. Then $m_{2}=n-2-b$. Denote by $t$ the number of tree components in $\mathcal{H}_{2}$. So, $t \geq b+2$. Next, denote by $i$ the number of isolated vertices in $\mathcal{H}_{2}$, by $j$ the number of isolated edges, by $k$ the number of stars with at least two leaves, and by $l$ the number of trees with diameter greater than two. Thus, $t=i+j+k+l$. We shall consider four subcases. 
Case 2A. There are at least two vertices of degree at least two in $\mathcal{H}_{2}$, and $j+k+l \geq 2$.

As above, we shall count, how many blocking edges have to be in $\mathcal{H}$. Denote by $n_{2}$ the number of vertices of degree at least two in $\mathcal{H}_{2}$. By assumption, $n_{2} \geq 2$. So, if we are not able to apply Lemma 5. we should have at least $\left(\frac{1}{2} i n_{2}\right) 3$-edges in $\mathcal{H}$. Similarly, if we are not able to apply Lemma $\sqrt{6}$, we should have at least $\left[\frac{1}{3} \cdot 4 \cdot\left(\begin{array}{c}j+k+l \\ 2\end{array}\right)\right]$ 3-edges in $\mathcal{H}$. Observe that one 3-edge can cover at most three pairs of vertices $x, y$ which satisfy the assumptions of Lemma 6 Moreover, between every two tree components with at least two leaves, there are at least four such pairs. There are $\left(\begin{array}{c}j+k+l \\ 2\end{array}\right)$ such pairs. Observe that all 3-edges mentioned above have to be distinct. Hence, we have

$$
\frac{1}{2} i n_{2}+\frac{4}{3} \cdot\left(\begin{array}{c}
j+k+l \\
2
\end{array}\right) \leq b \leq t-2=i+j+k+l-2
$$

Observe that

$$
\frac{1}{2} i n_{2} \geq i
$$

and

$$
\frac{4}{3} \cdot \frac{1}{2} \cdot(j+k+l)(j+k+l-1) \geq 1 \cdot 1 \cdot(j+k+l-1) .
$$

Again, we obtain a contradiction.

Case 2B. There are at least two vertices of degree at least two in $\mathcal{H}_{2}$, and $j+k+l<2$.

Thus, we have $l \leq 1$ and $n_{2} \geq 2$. Analogously as in Case $2 \mathrm{~A}$, we consider blocking edges in $\mathcal{H}$. If $l=0$, we obtain two cases:

1) if $j+k=0$, then

$$
i \leq \frac{1}{2} i n_{2} \leq b \leq t-2=i-2
$$

2) if $j+k=1$, then

$$
i \leq \frac{1}{2} i n_{2} \leq b \leq t-2=i-1 .
$$

If $l=1$ we have at least one blocking edge more. Then,

$$
i+1 \leq \frac{1}{2} i n_{2}+1 \leq b \leq t-2=i+l-2=i-1
$$

In all cases we get a contradiction.

Case 2C. There is at most one vertex of degree at least two in $\mathcal{H}_{2}$, and $j+k+l<2$.

By definition, $l=0$. Therefore, we have three subcases to consider. If $k=j=0$ or $k=0$ and $j=1$, then by Lemma 7, our claim is true. Thus, let $j=0$ and $k=1$. So, $\mathcal{H}_{2}$ consists of a star $K_{1, p}$ and $i$ isolated vertices. Observe that if $p \leq \frac{n}{2}$, then we are done by Lemma 7

Hence, let $p>\frac{n}{2}$. Then, $n=i+p+1$. Let $y$ be the center of the star, and let $x$ be an isolated vertex in $\mathcal{H}_{2}$. If for any vertex $z$, the set $\{x, y, z\}$ is not an edge of $\mathcal{H}$, then we are done by Lemma 5 . 
If the vertex $y$ belongs to two edges of the form $\{x, y, z\} \in \mathcal{E}(\mathcal{H})$ for any isolated vertex $x$, then we have the inequality

$$
p+2 \cdot \frac{i}{2} \leq n-2 .
$$

Since $p+2 \cdot \frac{i}{2}=n-1$, we obtain a contradiction.

Therefore, there exists an isolated vertex $x$ such that $\mathcal{H}$ contains exactly one 3 -edge $\{x, y, z\}$. Now, we construct a hypergraph $\mathcal{H}^{\prime}=\left(V^{\prime}, \mathcal{E}^{\prime}\right)$ such that $V^{\prime}=V-\{x, y\}$ and the set of edges is obtained from $\mathcal{E}$ as follows: we delete all 2-edges as well as the edge $\{x, y, z\}$, and we replace all remaining edges containing $x$ or $y$ (or $x$ and $y$ ) by new edges with these vertices deleted. Then $\mathcal{H}^{\prime}$ has two vertices less, and at least $p+1$ edges less than $\mathcal{H}$.

We shall show that there exists a packing permutation $\sigma^{\prime}$ of $\mathcal{H}^{\prime}$ without fixed points.

By the choice of $x$ and $y$ and the property of $\sigma^{\prime}$, it is easy to see that the permutation $\sigma=\sigma^{\prime} \circ(x y)$, where $(x y)$ denotes a transposition, will be a packing permutation of $\mathcal{H}$.

An edge of the form $\{x, s, t\} \in \mathcal{H}$ (where $s \neq y$ and $t \neq y$ ) will be called an $x$-edge. Analogously, an edge of the form $\{y, s, t\} \in \mathcal{H}$ (where $s \neq x$ and $t \neq x$ ) will be called a $y$-edge.

First, we consider the case where $\mathcal{H}$ has either $x$-edges or $y$-edges. We construct the hypergraph $\mathcal{H}^{\prime \prime}=\left(V^{\prime \prime}, \mathcal{E}^{\prime \prime}\right)$ as follows: $V^{\prime \prime}=V^{\prime}$, and the set of edges is obtained from $\mathcal{E}^{\prime}$ by deleting all $x$-edges and $y$-edges. So $m_{2}^{\prime \prime}=0$ in $\mathcal{H}^{\prime \prime}$. Now, we use a probabilistic argument as in the proof of Lemma7

$$
\operatorname{Pr}\left(\bigcup_{e, f \in \mathcal{H}^{\prime \prime}} A(e \curvearrowright f)\right) \leq\left(\begin{array}{l}
n \\
3
\end{array}\right)^{-1}(n-2-p-1)^{2} \leq \frac{6(n-6)^{2}}{4(n-2)(n-3)(n-4)}<\frac{1}{e}-\frac{1}{n !} .
$$

It is easy to observe that the last inequality holds for $n \geq 6$. (Recall that the probability that a random permutation has no fixed point is greater than or equal to $\frac{1}{e}-\frac{1}{n !}$.)

Now, suppose that there are $\xi x$-edges and $\eta y$-edges in $\mathcal{H}$. Observe that we have at least $p+3$ edges in $\mathcal{H}$ (there are $p$ edges of the star, the edge $\{x, y, z\}$, at least one $x$-edge and at least one $y$-edge). Then, $p+3 \leq n-2$. But $p>\frac{n}{2}$, hence $n \geq 11$. In general, we have at least $(\xi+\eta+1+p)$ edges in $\mathcal{H}$. Therefore $\xi+\eta \leq \frac{n}{2}-3$. Then a product $\xi \eta$ is maximal if $\xi=\eta=\frac{1}{2}\left(\frac{n}{2}-3\right)$. Analogously as above, we use a probabilistic argument to show that there is a packing permutation $\sigma^{\prime}$ without fixed points of $\mathcal{H}^{\prime}$. Observe that there are $\xi+\eta$ edges in $\mathcal{H}^{\prime}{ }_{2}$, and an $x$-edge cannot be mapped by $\sigma^{\prime}$ onto a $y$-edge (and vice versa). We have

$$
\begin{gathered}
\operatorname{Pr}\left(\bigcup_{e, f \in \mathcal{H}^{\prime}} A(e \curvearrowright f)\right) \leq \frac{2 \cdot 2 \xi \eta \cdot(n-2) !}{n !}+\left(\begin{array}{c}
n \\
3
\end{array}\right)^{-1}(n-2-p-3)^{2} \leq \\
\leq \frac{(n-6)^{2}}{4 n(n-1)}+\frac{3(n-10)^{2}}{2(n-2)(n-3)(n-4)}<\frac{1}{e}-\frac{1}{n !} .
\end{gathered}
$$

It is easy to check that the last inequality is satisfied for $n \geq 11$, and consequently, there exists a packing permutation of $\mathcal{H}^{\prime}$ without fixed points. 
Case 2D. There is at most one vertex of degree at least two in $\mathcal{H}_{2}$, and $j+k+l \geq 2$.

Then, $\mathcal{H}_{2}$ has only tree components, $l=0$ and $k \leq 1$.

If $k=0$, then $j \geq 2$ and $j \leq \frac{n}{2}$ (because $j$ is the number of isolated edges in $\mathcal{H}_{2}$ ). Then, by Lemma 7 , the conclusion holds.

Thus, let $k=1$ and $j \geq 1$. Denote by $K_{1, p}$ the star in $\mathcal{H}_{2}$. If $p+j \leq \frac{n}{2}$, we are done by Lemma 7 . Hence $p+j>\frac{n}{2}$ and $n=i+2 j+p+1$. If $j=1$, then a 3 -edge can block at most two possibilities for the choice of two leaves in $\mathcal{H}_{2}$ if one leaf is in the star. So, if we are not able to apply Lemma6, we have to have at least $\frac{2 p}{2}$ blocking edges in $\mathcal{H}$. If we are not able to apply Lemma 5 , we have to have at least $\frac{i}{2}$ blocking edges in $\mathcal{H}$. Observe that in both cases the blocking edges are distinct. Hence, taking into account all 2-edges we get

$$
n-2 \geq|\mathcal{E}| \geq p+1+p+\frac{i}{2}
$$

and

$$
n-3 \geq 2 p+\frac{i}{2}
$$

On the other hand, $n-3=i+p$. Therefore, $\frac{i}{2} \geq p$. So, $n-3 \geq 3 p$. It follows that $p<\frac{n}{3}$, a contradiction.

Now, let $j \geq 2$. Observe that the number of 3-edges in $\mathcal{H}$ is at least $\frac{i}{2}$ (because of Lemma 5 , and at least $\frac{2 p j}{2}$ (because of Lemma 6. (In the latter case, we may assume that one of the leaves comes from the star.)

We have

$$
n-2 \geq|\mathcal{E}| \geq j+p+p j+\frac{i}{2}
$$

But $j+p>\frac{n}{2}$, so

$$
n-2 \geq \frac{n}{2}+p j+\frac{i}{2}
$$

Hence

$$
\frac{n}{2}-\frac{i}{2}-2 \geq p j
$$

We know from a structure of the hypergraph that $n=i+2 j+p+1$, so it follows from the above inequality that

$$
\frac{2 j+p+1-4}{2} \geq p j
$$

This inequality together with the fact that $2 p j \geq 2 p+2 j$ for $p, j \geq 2$, implies

$$
2 j+p-3 \geq 2 p j \geq 2 p+2 j,
$$

a contradiction.

This ends the proof of the theorem. 


\section{References}

[1] A. Benhocine, A. P. Wojda, On self-complementation, J. Graph Theory 8 (1985), 335-341.

[2] B. Bollobás, Extremal Graph Theory, Academic Press, London (1978).

[3] D. Burns, S. Schuster, Every $(n, n-2)$ graph is contained in its complement, J. Graph Theory 1 (1977), 277-279.

[4] M. Pilśniak, M. Woźniak, A note on packing of two copies of a hypergraph, Discussiones Math. G. Th. 27(1) (2007), 45-49.

[5] M. Woźniak, Embedding graphs of small size, Discrete Applied Math. 51 (1994), 233-241.

[6] M. Woźniak, Packing of graphs, Dissertationes Math. 362 (1997), 1-78.

[7] M. Woźniak, Packing of graphs and permutations - a survey, Discrete Math. 276 (2004), 379-391.

[8] H. P. Yap, Some Topics in Graph Theory, London Math. Society, Lecture Notes Series, Vol. 108, Cambridge University Press, Cambridge, 1986.

[9] H. P. Yap, Packing of graphs - a survey, Discrete Math. 72 (1988), 395-404. 